City University of New York (CUNY)

CUNY Academic Works

\title{
The Mathematics and Applications behind Image Warping and Morphing
}

Tanvir Prince

CUNY Hostos Community College

Maria Malik

Princeton University

Ildefonso Salva

Mott Haven Village Preparatory High School

Ariel Mazor

CUNY Borough of Manhattan Community College

Sakhr Aldaylam

CUNY Hostos Community College

\section{How does access to this work benefit you? Let us know!}

More information about this work at: https://academicworks.cuny.edu/ho_pubs/77

Discover additional works at: https://academicworks.cuny.edu

This work is made publicly available by the City University of New York (CUNY).

Contact: AcademicWorks@cuny.edu 


\title{
The Mathematics and Applications behind Image Warping and Morphing
}

\author{
Tanvir Prince ${ }^{1}$, Maria Malik ${ }^{2}$, Ildefonso Salva ${ }^{3}$, Ariel Mazor $^{4} \&$ Sakhr Aldaylam ${ }^{5}$ \\ ${ }^{1}$ Department of Mathematics, City University of New York, Bronx, NY, USA \\ ${ }^{2}$ The Urban Assembly School for Criminal Justice, Princeton University, Brooklyn, NY, USA \\ ${ }^{3}$ Department of Mathematics, Mott Haven Village Preparatory High School, Bronx, NY, USA \\ ${ }^{4}$ Department of Mathematics, Borough of Manhattan Community College, Manhattan, NY, USA \\ ${ }^{5}$ Department of Engineering, The City University of New York, Bronx, NY, USA \\ Correspondence: Tanvir Prince, Associate Professor, Department of Mathematics, Eugenio María de Hostos Community \\ College of the City University of New York, Bronx, NY, USA. E-mail: tprince @ hostos.cuny.edu
}

\author{
Received: October 21, 2015 Accepted: November 9, 2015 Online Published: November 25, 2015 \\ doi:10.5539/jmr.v7n4p167 \\ URL: http://dx.doi.org/10.5539/jmr.v7n4p167
}

\begin{abstract}
This research is conducted in the summer of 2015 and is possible by the support of various agency, in particular, by the grant of Prof. Angulo Nieves and the New York City Research Initiative.

The purpose of this research is to reveal the mathematics and applications of the computer animation techniques of warping and morphing. A warp is a twist or distortion in the form of an object in an image while a morph is the smooth and gradual transformation of an object in one image into the object in another image. Linear algebra makes these computer animation techniques possible; the first phase of this research delves into how those mathematical processes translate into image warps and morphs. Image morphs and morphs were identified as affine transformations of original images. The second part of this study requires the analysis and application of image warping and morphing techniques in an array of fields. The team utilized the computer software, Abrosoft Fantamorph and Morpheus in order to create a series of warps and morphs. This shows an example of the use of technology in undergraduate research. The final phase of this research was to identify what uses NASA can have for these computer animation techniques and what further research can be done to expand our knowledge of warps and morphs. By identifying the mechanics of warps and morph, we can discover how they can assist scientists and organization, such as NASA, to create depictions of objects, ideas, places, and events. Ultimately, studying morphing and warping techniques allows us to find better ways to represent visual data whether it is images of the ozone hole or maps of the ever-changing weather in a region. The limitations that were found during the study can be used to conduct further research about warps and morphs - such as distorting images using quadratics or varying the rate at which each part of a transformation happens.
\end{abstract}

Keywords: image warping, image morphing, affine transformation, NASA, Abrosoft Fantamorph

\section{Introduction}

Have you ever wondered about how the 3D representations of the moon are created? Or how images from different sources - especially satellites - are combined to form uniform and cohesive images? Or even how the gradual and slow process of a person aging can be depicted from just two images? Such are the questions that can be answered as a result of an in depth analysis of warping and morphing techniques and the mathematics and applications behind them.

In definition, warps and morphs are computer animation techniques that depict the distortion, manipulation, or transformation of images. To be more specific, a warp is a twist in the form or shape of an image. An example of this is distorting the image of a chicken by elongating its legs. Warping two complementary objects by forcing them to blend and assume the form of one another results in a morph - which is the smooth and gradual transformation of the image of one object into that of another. An example of a morph is transforming the image of a chicken into the image of a dinosaur. One of the biggest differences between a warp and a morph is that while a warp only requires one picture, a morph requires at least two pictures - to create longer and more complicated morphs, more images are necessary. The mathematics behind these computer animation techniques will be discussed later in the research, however, it is important to note that the process of a warp or morph is purely dependent on linear algebra.

In essence, a morph is two warps happening at the same time, in a synchronized fashion. The beginning image is warped with respect to the end image and vice versa. An example showing the mechanics of a morph is shown below: 

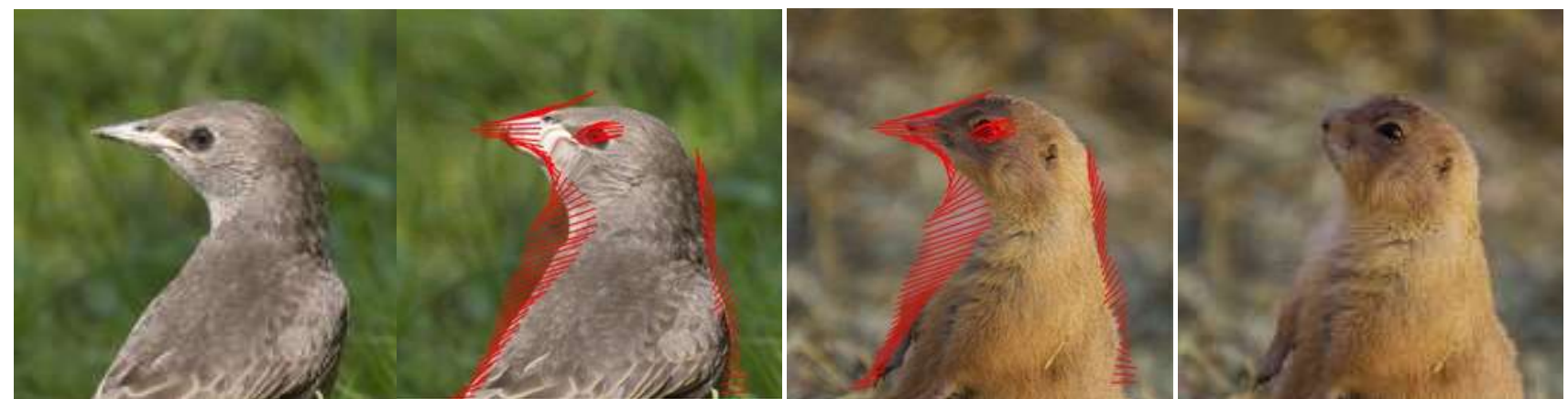

Figure 1.1. Mechanics of a Morph [3]

Both images are manipulated so that each is warped in the direction of the other. As the objects in the images assume a different form, they are overlapped and their color densities are averaged in accordance to the time. The example shown above follows the morphing process of a bird as it transforms into a prairie dog. The red shaded area in the second image indicates the change in its shape that the bird had to undergo in order to morph into the prairie dog. A similar process is happening in the opposite direction; the prairie dog's form is altered so that it assumes the shape of the bird. While this shape transformation is happening, the color densities of corresponding pixel are averaged in a specific way. For example, assuming the morph happens within the time frame of $\mathrm{t}=0$ and $\mathrm{t}=1$, at $\mathrm{t}=0.25$, the color density of the first picture would account for three-fourths of the colors in that specific frame, while the color density of the second picture would only account for one-fourth of the color in the frame. The color densities of the rest of the frames in the morph would be calculated in the same way.

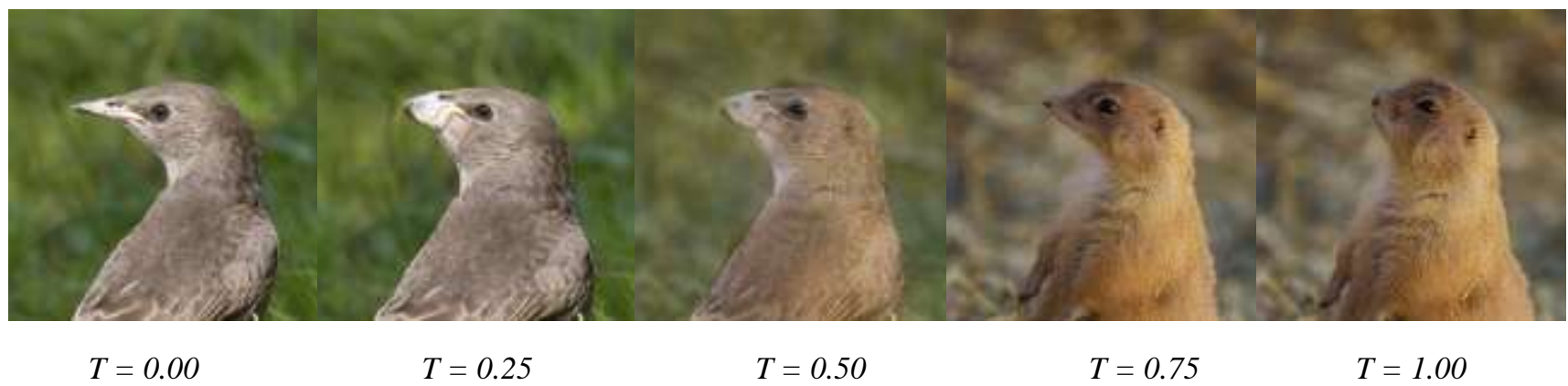

Figure 1.2. Time Frame of Averaging Picture Densities

The processes of warps and morphs can be instigated by using specially-designed computer software. The method that is used to create the specific warps and morphs described above entails a linear process in which the rates at which the objects in the images change are constant. The mathematics and applications behind warps and morphs reinstate how incredibly useful they can be.

\section{The History of Image Warping and Morphing}

The term "morphing" is derived from the biological phrase "metamorphosis," which refers to the change in bodily form that an organism experiences during its growth and development process. In this way, the computer animation technique of morphing is very similar to biological metamorphosis: one object in an image is transformed into an object in another image. The word "warp" originates from the Old English term "weorpan" - meaning "to throw" or "fling open." Likewise, an image warp is generated when the object in one image is distorted in a way such that it seems as if parts of the original form of the object have been thrown in different directions. The form of the object is twisted and "flung open" so that it assumes a new form.

For decades, the entertainment industry has used various methods of image warping and morphing to enhance the visual effects of film. Advanced computer software has made it easier to warp/morph objects in order to create special effects and make scenes in movies more visually attractive. In fact, the use of modern morphing techniques can be traced back to the 70s. Though computer-animated morphing/warping techniques have been employed for decades in the film industry, these techniques can be utilized in other ways - from depicting the growth of a tumor to illustrating gradual environmental changes. Today, various versions of morphing/warping software have found their way into the market. Some of the more prominent examples include Abrosoft Fantamorph, Morpheus Photo Morpher, Angelmorph, and FaceMorpher. 


\section{Methods}

The team used a software called Abrosoft Fantamorph 5.0 to experiment and create a series of image warps and morphs. Previous versions of this software exist, but the 5.0 version is more efficient and easier to use. Some of the applications of warping and morphing techniques included in this paper were created using another image warping/morphing software called Morpheus. Like Abrosoft Fantamorph, Morpheus allows for point to point correspondences on two images and has features that depict the process of triangulation during a warp or morph. However, unlike Abrosoft Fantamorph, Morpheus is somewhat more challenging to navigate. The Delux version of Abrosoft Fantamorph is especially comprised of a greater array of unique features. These include (but not limited to): creating a compilations of different warps and morphs, changing the amount of frames it takes for a transformation, viewing points, lines and triangles, and more. The software utilizes mathematical means - such as triangulation - in order to apply warp/morph techniques such as generating smooth transitions between different images and creating distortions in the forms of objects in selected images.

The warps and morphs created by the software were generally generated in a linear fashion and contained a constant rate of transformation. The team used the Deluxe version of the software in order view the process of triangulation as the images were warped or morphed. The left picture in figure 1.3 below is a screenshot of the typical layout of Abrosoft Fantamorph when one is creating a morph using the 5.0 version on windows 8.0. Notice that there are three distinct boxes in the standard software layout, each with a specific purpose: the top left hand box for image 1, the top right hand box for image 2 , and the bottom box to depict the transition from image 1 to image 2 . The software can also adjust the images - by cropping - to ensure that the images can easy correspond and will result in a smoother warp or morph. The right picture in figure 1.3 is a sample sequence route of the morph.
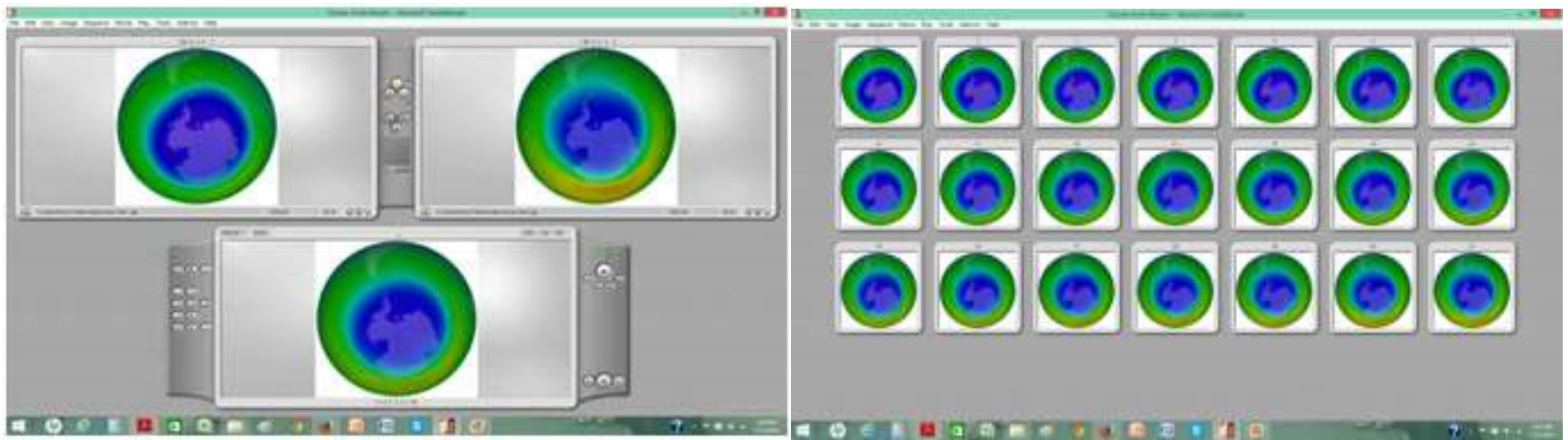

Figure 1.3. Abrosoft Fantamorph Window and Frame Sequence

\section{Procedures}

\section{A. Procedure to Create a Warp:}

1. The first part in the process of creating an image warp is to input points at key features of the image that will be warped, hence known as "image 1." Key features are any defining parts of the image that make the specific image unique. For example, in an image depicting a human face, key features can include the eyes, lips, nose, and hairline. As many points as possible should be added to the key features in order to create a smoother warp. Additional points can also be added in other parts of the image in order to stabilize the end result (the final warped image.) The process of adding points to the image is called "establishing image correspondences."

- Make sure to add points at the edges and corners of the image to form a rectangular region.

2. At this point, after as many points have been inserted on the key features of image 1, the computer software generates a triangular mesh over image 1 and a corresponding mesh over the end image that will be the product of the warp, henceforth known as image 2. These facts should be noted:

- At this moment, since the triangular mesh over both images is congruent, the images are also congruent. A warp is only created when the points in image 2 are moved to new destinations.

- Each point is a vertex of as many triangles that can be formed from the surrounding points as possible. The point-to-point correspondences ultimately translate into triangle-to-triangle correspondences over image 1 and image 2 .

3. The last step is to move certain and specific points in image 2 into new locations. Through an affine transformation, each point is separately mapped from the first picture (the source) to the second picture (the destination). 


\section{B. Procedure to Create A Morph:}

***Because image morphs are very similar to image warps, the procedure of an image warp will be very similar to the procedure of an image morph.

1. The first part in the process of creating an image morph is to input points at key features of the image that will be warped, hence known as "image 1." Key features are any defining parts of the image that make the specific image unique. For example, in an image depicting a human face, key features can include the eyes, lips, nose, and hairline. As many points as possible should be added to the key features in order to create a smoother warp. Additional points can also be added in other parts of the image in order to stabilize the end result (the final warped image.) The process of adding points to the image is called "establishing image correspondences."

- Make sure to add points at the edges and corners of the image to form a rectangular region.

2. At this point, after as many points have been inserted on the key features of image 1, the computer software generates a triangular mesh over image 1 and a corresponding mesh over the end image that will be the product of the warp, henceforth known as image 2 . The key points of image 1 are overlaying image 2 in the exact coordinates they are located in image 1. These facts should be noted:

- At this moment, since the triangular mesh over both images is congruent, the images are also congruent. A warp is only created when the points in image 2 are moved to new destinations.

- Each point is a vertex of as many triangles that can be formed from the surrounding points as possible. The point-to-point correspondences ultimately translate into triangle-to-triangle correspondences over image 1 and image 2 .

3. Move points in image 2 to corresponding key features in order to create correspondences between specific key features. For example, if points $\mathrm{w}_{1}, \mathrm{x}_{1}, \mathrm{y}_{1}$, and $\mathrm{z}_{1}$ are positioned around the pupil of the image of an human eye in image 1 , then one must move points $\mathrm{w}_{2}, \mathrm{x}_{2}, \mathrm{y}_{2}$, and $\mathrm{z}_{2}$ (the mapped points on image 2 ) to the new corresponding locations - the pupil of a tiger eye, for example - in image 2.

- Avoid placing points in locations that cause overlapping triangles in image 2. If this were to happen, there would be lapses and distortions in the otherwise smooth morph between image 1 and image 2.

\section{Applications of Image Warping and Morphing}

Through image warping and morphing is primarily utilized by the film industry, the team found that these computer animation techniques have profound uses in other fields as well. For instance, image morphs can be used to map the gradual deforestation of a rain forest between a specific time frame and can also be utilized to view the potential evolutionary path an organism might have undergone in order to become the species it is in the present. Furthermore, image morphs can be used to create visual representations of things like the growth of a cancer tumor or the decline of harmful bacteria in an individual's stomach. In addition to applications in the environmental and life sciences, image warping and morphing techniques can also be applied to space science, face recognition technologies, and the creation of 3D models of objects, such as planets, human bodies, plants, and more. In particular, generating 3D models from 2D images can be extremely useful in the process of creating cohesive 360 degree representations of an object. The team applied warping and morphing techniques to many images from an array of fields. Outlined below are some of those major applications.

\section{A. Applications in the Environmental Sciences:}

\section{Air Pollution}
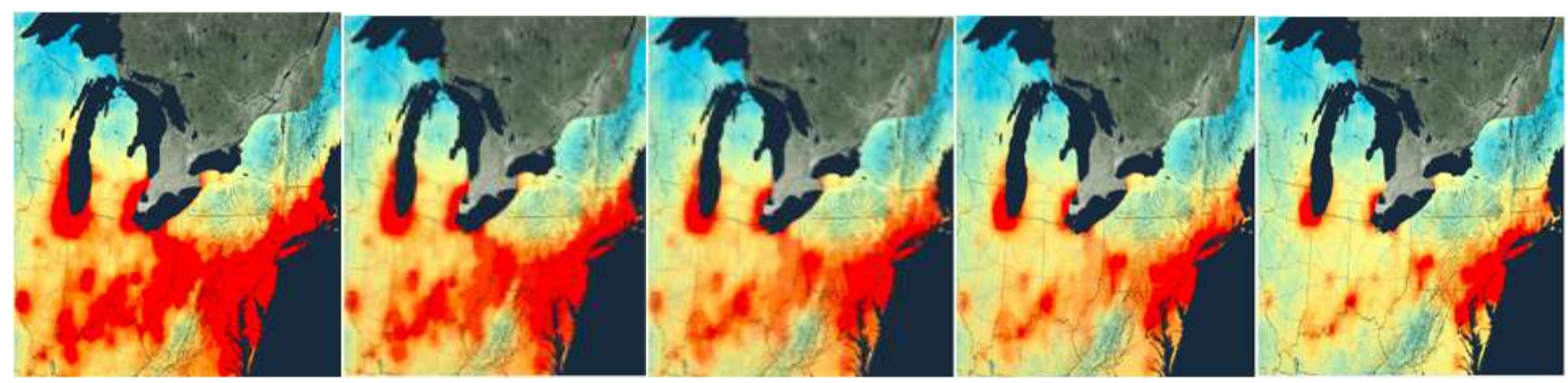

Figure 1.4. Air Pollution and $\mathrm{NO}_{2}$ Concentrations Morph [Schindler, 2014] 
ABOVE: Image warping and morphing is useful in mapping environmental concerns of the, such as air pollution. In this series of frames (extracted from a morph), it is possible to see the decrease of $\mathrm{NO}_{2}$ in at the Northeastern region of the United State. The maps show the gradual decline of $\mathrm{NO}_{2}$ between 2005 and 2011 [Schindler, 2014]. By creating a morph of this change, it becomes easier to analyze the extent air pollution or atmospheric gases over a specific region.

\section{Ozone Hole}
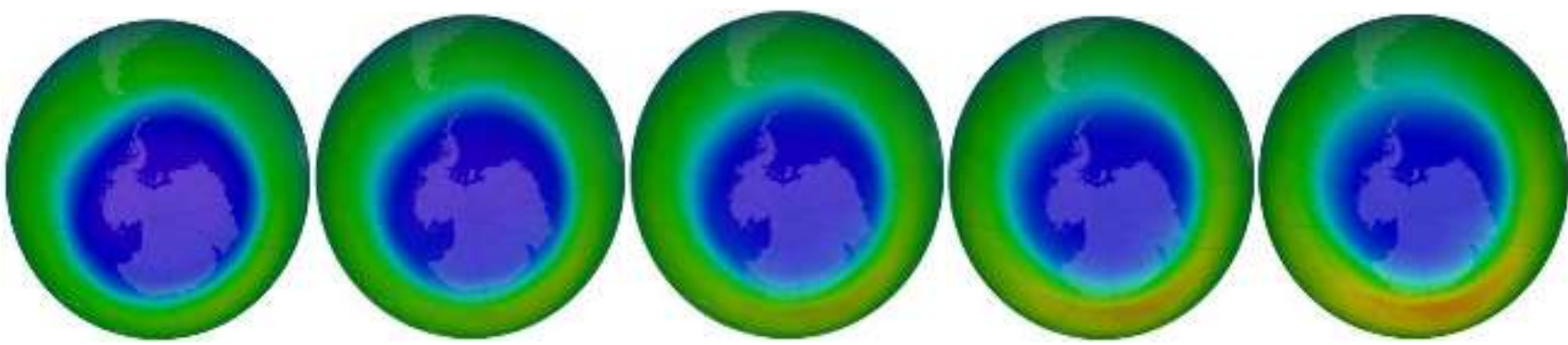

Figure 1.5. Ozone Hole Morph [Climate Discovery, 2013]

ABOVE: Some changes in a vector are hard to view with the naked eye. Morphing images that depict slight changes can create better and easier-to-view depictions of those vectors. In the frames shown above, morphing software has been used to illustrate the slight decrease in the size of the ozone hole (shown in blue) from September 2006 to September 2012 [Climate Discovery, 2013]. Despite the fact that this decrease is not very noticeable in separate images, morphing those images creates a more successful, more apparent comparison.

\section{Arctic Sea Ice}
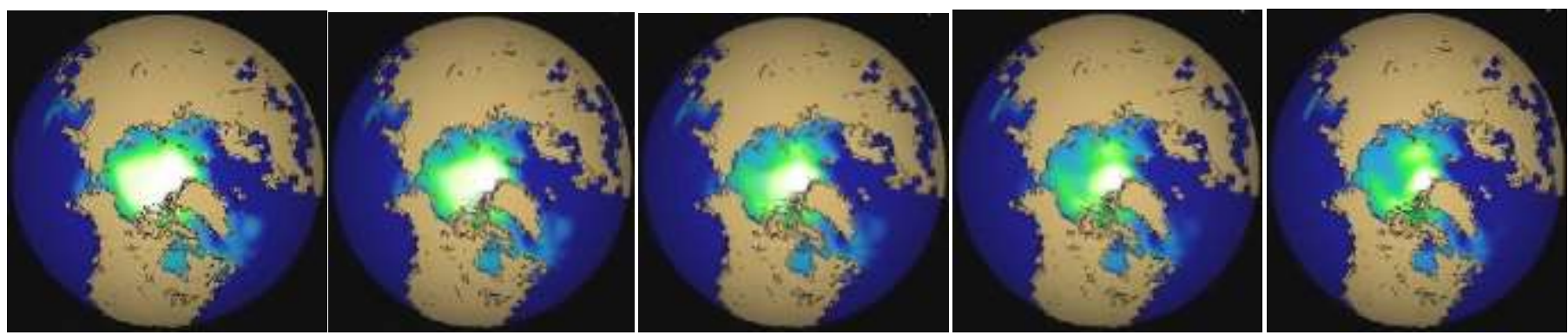

Figure 1.6. Arctic Sea Ice Morph [Laden, 2011]

ABOVE: Through the use of the image morphing software, it is possible to create a comparison depicting the gradual changes in sea ice thickness. The above comparison shows the dramatic decrease of sea ice thickness in the Arctic region from the 1950s to its projected thickness in 2050 (this estimate is based on reasonable assumptions of climate change) [Laden, 2011]. In fact, more recent analyses suggest that this projection might actually be optimistic. The white area shows the thicker sea ice and the green depicts thinner ice.

\section{California Drought Map}
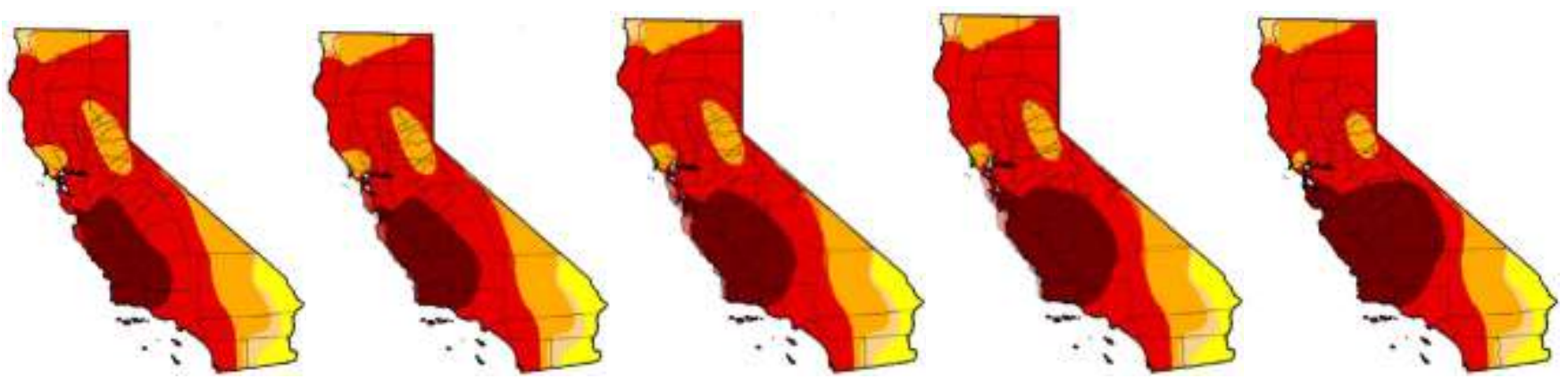

Figure 1.7. California Drought Morph [Park, et al., 2014]

ABOVE: Image morphing is also useful in mapping the extent of natural disasters, such as droughts. In this series of frames (extracted from a morph), it is possible to see the rate of change of drought intensity in California over a one week span [Park, et al., 2014]. The dark red in the map depicts the areas with the highest drought intensity. By creating a morph 
of this change, it becomes easier to analyze the extent and intensity of a drought - or any other natural disaster for that matter - on a given piece of land.

\section{Effect of California's Drought on Lake Oroville}

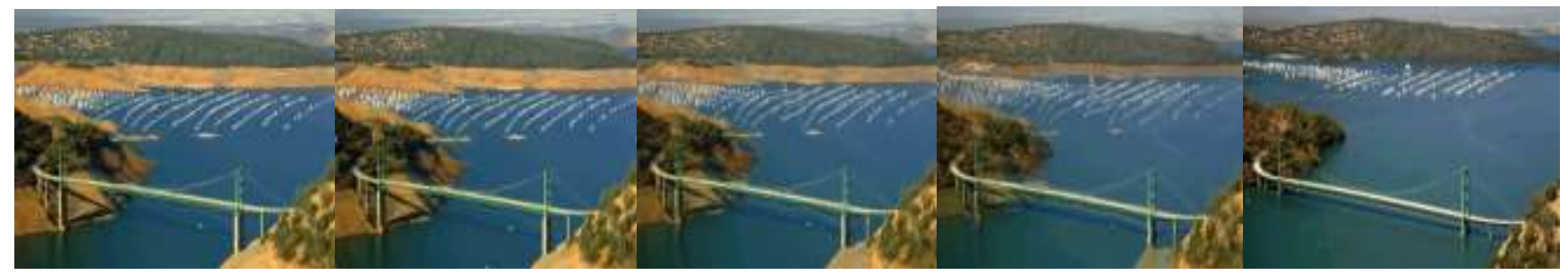

Figure 1.8. Lake Oroville Drought Morph [8]

ABOVE: This is an image morph of 2 images that depict the change in Lake Oroville's sea level between July 16,2011 and Jan. 16, 2014 [Spector, 2014]. Morphing techniques can be utilized in order to illustrate changes in landscapes due to natural disasters, including drought. The change shown in the image sequence above shows a dramatic decline in the sea level of Lake Oroville. This information can be used in educational campaigns to inform individuals of the effects of the drought and to encourage water conservation efforts.

\section{Population Growth Maps}

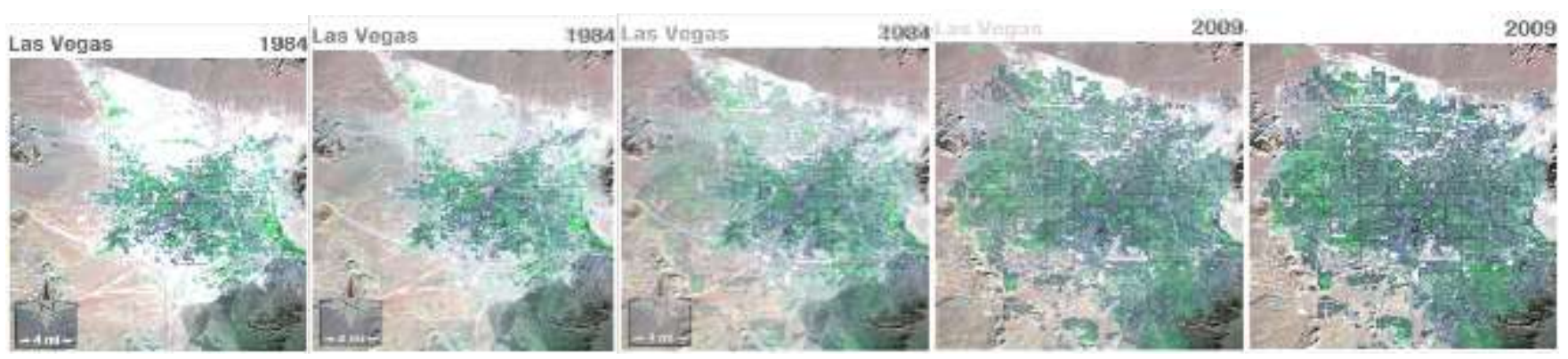

Figure 1.9. Urban Growth Morph [9]

ABOVE: The image sequence above depicts the population growth of the city of Las Vegas between the years 1984 and 2009. The morphing process can be used as a model to predict future growth of various cities by serving as a tool that predicts rates of change. Morphing techniques can allow scientists to measure population growth in various regions with minimal data. In this example, only two images of Las Vegas were utilized to generate the morphed model that shows the rapid growth of the city. The image right in between the sequence can provide a rough estimate of how much the city had expanded in the year 1996.

\section{B. Applications in Space Science:}

\section{Super Nova Morph}
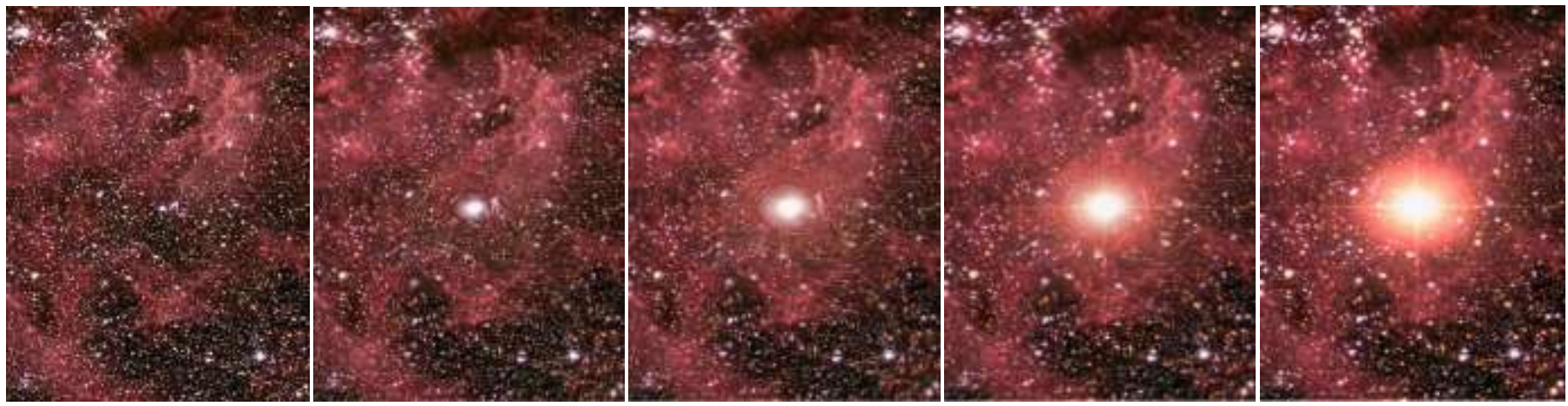

Figure 2.0. Large Magellanic Cloud Super Nova Morph [NASA, 2013]

ABOVE: On February 23th, 1987, a star exploded in the Large Magellanic Cloud - a nearby galaxy. NASA was able to obtain images of the supernova before and after its explosion [10]. The frames from a morph that depicts the explosion of the star are shown above. Through image morphing technologies, it becomes possible to view the course a star takes as it 
explodes. Though the morph created here assumes a constant rate of explosion, other non-linear morphs can remedy this and provide more accurate representations.

\section{Applications in the Medical Field:}

1. Cancer Cell

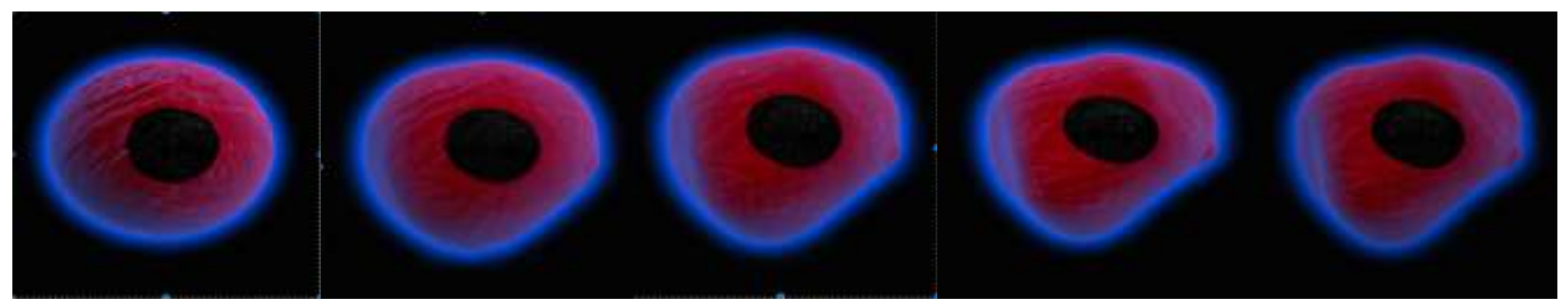

Figure 2.1. Normal Cell to Cancer Cell Morph [imgLOP, 2015]

ABOVE: Image morphing techniques can also be utilized to create representations of natural life processes or organisms. In the image sequence above, a normal animal cell is transformed into a cancer cell [imgLOP, 2015]. The process in between the cell phases can be replicated by morphing images of the normal cell and cancer cell together. Thus, scientists and doctors can easily visualize the path cells take in order to become tumors. Furthermore, other biological processes can also be visualized using image morphing techniques [imgLOP, 2015]. For example, the growth of an animal can be mapped from its infancy to adulthood. In addition, the spread of various diseases in a body can be represented using morphing techniques in order to observe the sequences of how the diseases proliferated.

\section{Applications in Face-Recognition Technologies:}

\section{Human Morphs}

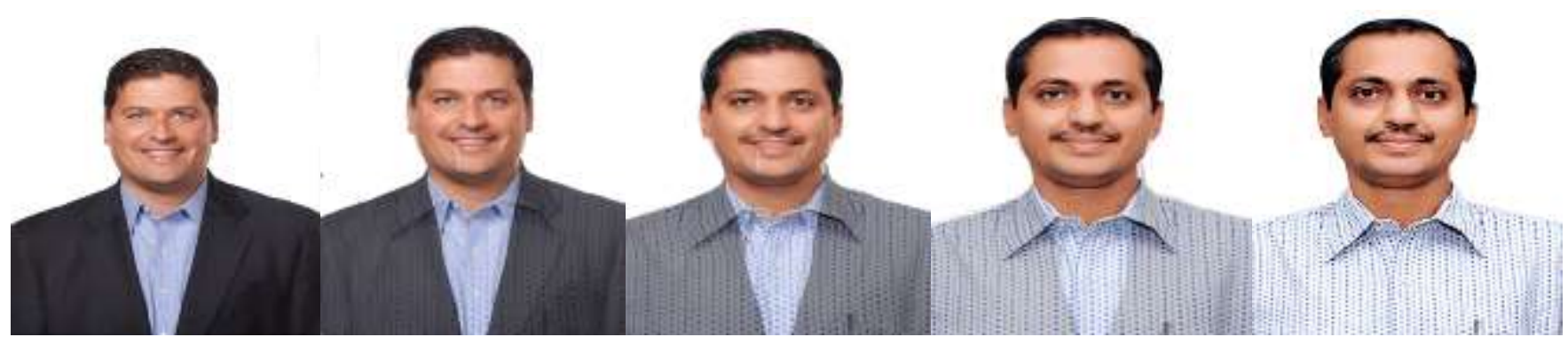

Figure 2.2. Facial Recognition Morph

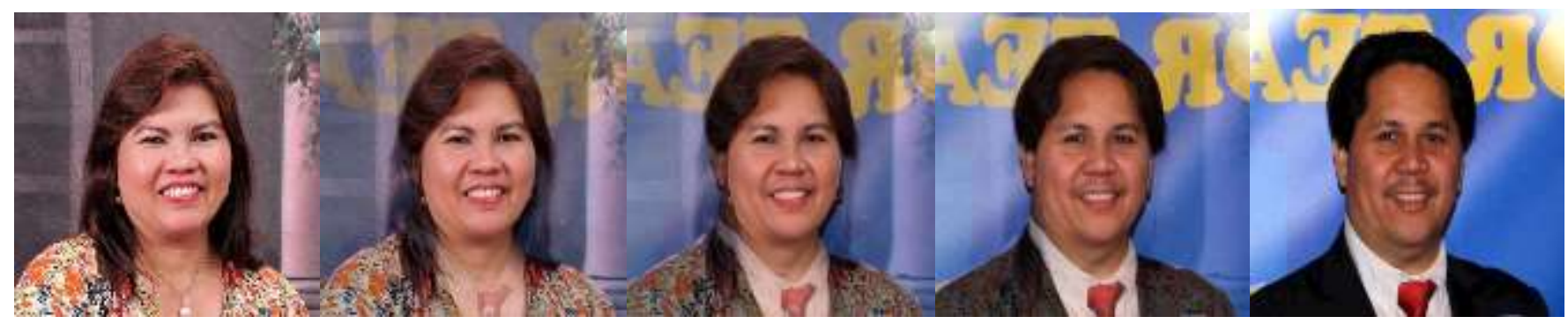

Figure 2.3. Comparing Facial Structure Differences

ABOVE: Morphing technologies are also popularly utilized to create morphs between different humans or to depict the gradual aging process of one human. Morphing family members - or any humans for that matter - can be used to compare similarities or differences in facial structure and body form. Facial recognition technologies can use morphing techniques in order to calculate how different the facial features of the individuals in two different images are in order to suggest if the images is of the same person. This technique can be used in the criminal justice field in order to identify possible perpetrators of the law. Furthermore, the aging process of one person can also be visually depicted from only two images. This can be used to show how an individual gradually aged in a specific time frame.

\section{E. Applications in 3D Model Generation:}

\section{3D Camera View of a Human}



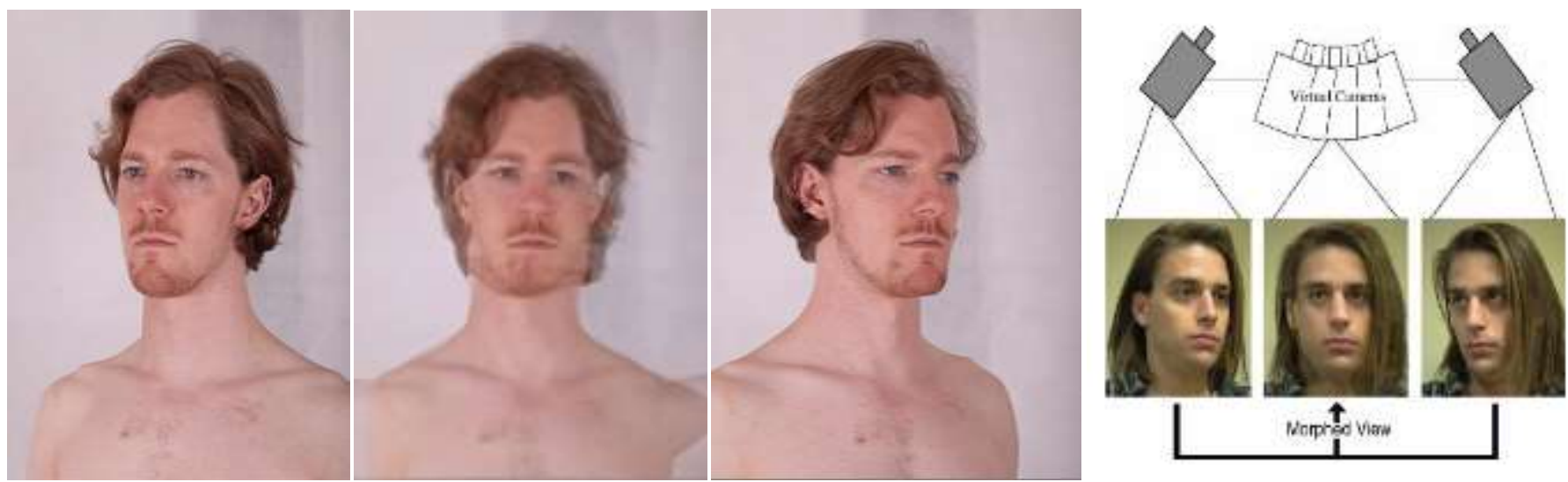

Figure 2.4. Camera Views and 3D Illusions

ABOVE: Another useful application of morphing software is the possibility to create 3D illusions that are reminiscent of utilizing a virtual camera. After taking images from various angles of an object, one can separately morph consecutive images. The next step is to collate these individual morphs. As a result, when observing the result - the "master" morph one is given the illusion of viewing a virtual camera. The 3-dimensional illusion is thus created by morphing 2-dimensional images. The images above show how a 3D camera view of a man's head can potentially look like. The middle picture has been created by morphing the left and right side images of the head. In animation form, one can see 120 degrees around the front side of the man's head. Though this 3D illusion is a compilation of 2D morphs, the viewer cannot easily differentiate if the morphs are seamlessly collated. This significant implication can be used in an array of fields; we can create virtual camera and 3D depictions of landforms, planets, humans, animals, objects, locations, inventions, machines, and more. For instance, videos from virtual cameras have too much memory that cannot be easily sent back to the Earth from far away locations in Space. In this circumstance, a series of smaller images - which take up a smaller amount of bytes - can be taken and sent back to Earth where they can be morphed to create a 3D "camera view" of an object, such as a planet.

\section{Mathematics behind Image Warps and Morphs (Overview)}

Computer graphics software allows for the manipulation of images in various ways - rotating, dilating, shearing, scaling, cropping, slanting, etc. Using linear algebra, computer software can also be developed to create warps and morphs. A summary of the math which is used for these techniques is outlined below.

Warps and morphs are examples of affine transformations. An affine transformation is any type of transformation that preserves the ratio of distances between the points and the collinearity of each of the points. In essence, any triangle is capable of being transformed into another triangle by the means of an affine transformation. As a result, all triangle are affine.

To create a simple warp, we must first construct a triangular region on a plane by choosing three noncollinear points, where [Anton, 2010]:

$$
V=C_{1} V_{1}+C_{2} V_{2}+C_{3} V_{3}
$$

This triangle from the first image is mapped on to a new plane, where [Anton, 2010]:

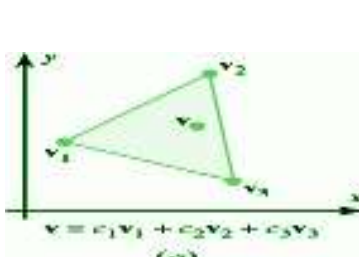

(ci)

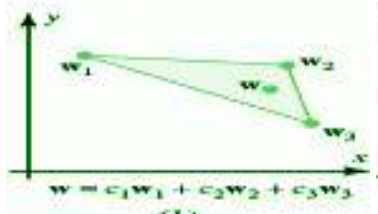

(b)
$W=C_{1} W_{1}+C_{2} W_{2}+C_{3} W_{3} \quad$ and:

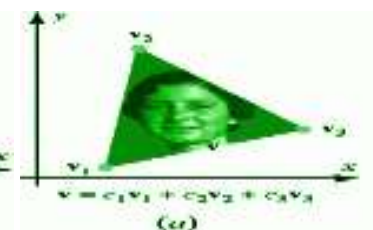

(e)

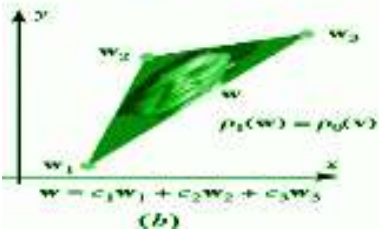

Figure 3.1. Triangulation and Image Mapping [Anton et al., 2010]

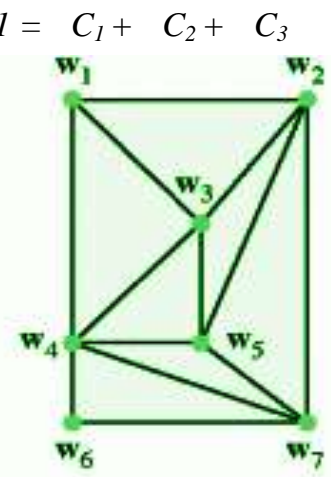

(b)

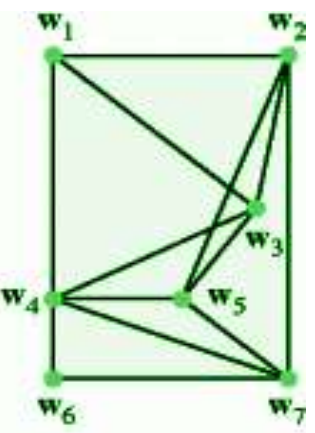

(c) 
As shown above in figure 3.1, each point in image 1 is mapped on to image 2 . The mapped points in image 2 are then moved to new locations. The linear process that a point takes from its location in image 1 to its new corresponding location in image 2 is an affine transformation. Specifically, in a procedure of a morph, points in a hypothetical image 2 are moved corresponding key features in order to create point correspondences between those specific key features in image 1 and image 2. For example, if points $w_{1}, x_{1}, y_{1}$, and $z_{1}$ are positioned around the pupil of the image of an human eye in image 1 , then points $\mathrm{w}_{2}, \mathrm{x}_{2}, \mathrm{y}_{2}$, and $\mathrm{z}_{2}$ (the mapped points on image 2) are moved to the new corresponding locations the pupil of a tiger eye, for example - in image 2 . The points, $\mathrm{w}_{1}, \mathrm{x}_{1}, \mathrm{y}_{1}$, and $\mathrm{z}_{1}$ move in a linear fashion and a constant rate between $t=0$ and $t=1$, so that in the end of the transformation, they assume the location and identities of points $w_{2}, x_{2}, y_{2}$, and $z_{2}$ (the mapped points on the second image). This short procedure is the general mathematical functioning behind a simple warp. Because a morph is two separate warps happening simultaneously in opposite directions, this entire mathematical process of point correspondences, triangulation, mapping, and interpolation is happening twice.

In the general equation of the warping process, $\mathrm{C}$ represents a unique constant. This constant, in turn, represents how each point in a warp must move to create an affine transformation. In order to generate a smooth and successful warp or morph, there are some critical conditions that must be satisfied during the triangulation of a specific vector (usually the triangulation of image 1) [Anton et al., 2010]:

1. The line segments form the sides of a set of triangles. [Anton et al., 2010]

2. The line segments do not intersect. [Anton et al., 2010]

3. Each vertex point is the vertex of at least one triangle. [Anton et al., 2010]

4. The union of the triangles is the rectangle. [Anton et al., 2010]

5. The set of triangles is maximal (i.e., no more vertices can be connected). [Anton et al., 2010]

Because a morph is two warps that are combined, the math behind warps is a major part of the math behind morphs. However, in a morph, the densities of the colors in the two images are also being averaged. The color density of any two corresponding points on the images that are being morphed is averaged by the following equation [Anton et al., 2010]:

$$
p_{t}(u)=(1-t) p_{0}(v)+t p_{1}(w)
$$

Where[Anton et al., 2010]: $\quad p_{l}(w)=$ End Picture's Density $\quad p_{0}(v)=$ Begin Picture's Density $\quad t=$ at any time, $t$

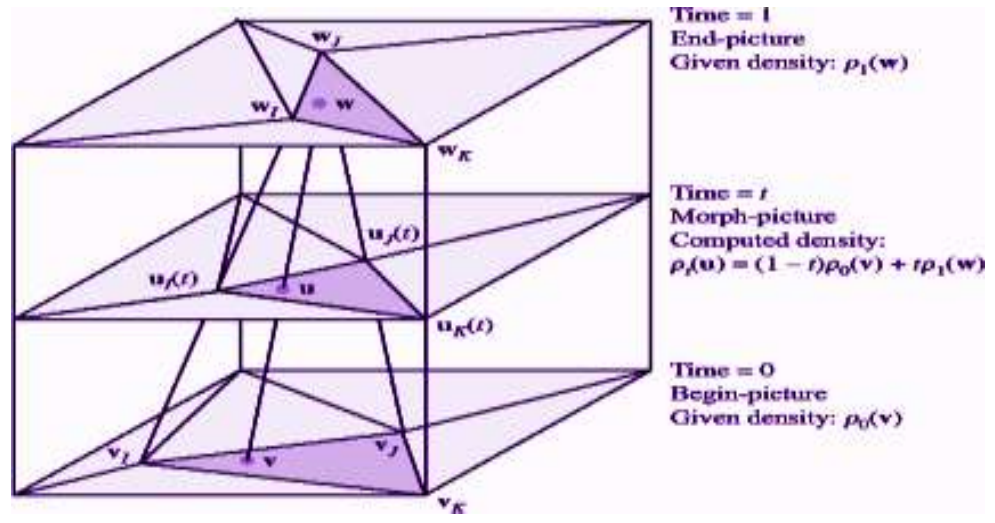

Figure 3.2. The Process of Averaging Picture Color Densities [Anton et al., 2010]

The main color sequence that is averaged is comprised of red, blue, and green (RBG) [15]. While the warping or shape transformation of two images is happening, the color densities of corresponding pixels are averaged in a specific mathematical way. For instance, assuming the morph happens within the time frame of $\mathrm{t}=0$ and $\mathrm{t}=1$, at $\mathrm{t}=0.25$, the color density of the first picture would account for three-fourths of the colors in that specific frame, while the color density of the second picture would only account for one-fourth of the color in the frame. The color densities of the rest of the frames in the morph would be calculated in the same way. As one views the animation of the final morph, one can see how the color densities of image 1 and image 2 are averaged accordingly to create a smoother transformation. Generally speaking, the mathematics behind image warps and morphs assume that the transformation occurs between $t=0$ and $t=1$, but it is definitely possible to instigate warps and morphs in a shorter or longer time frame. 

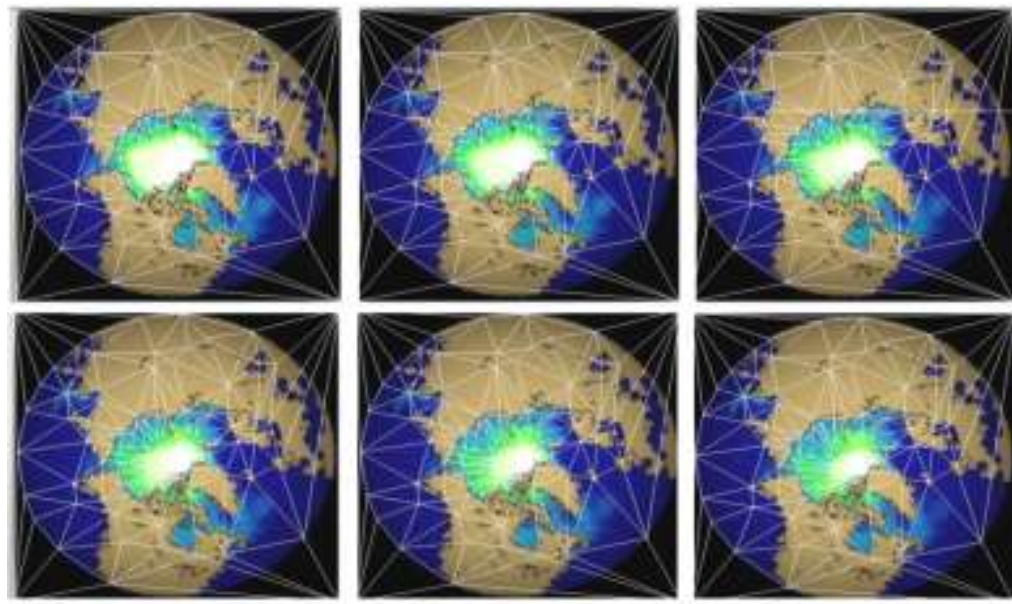

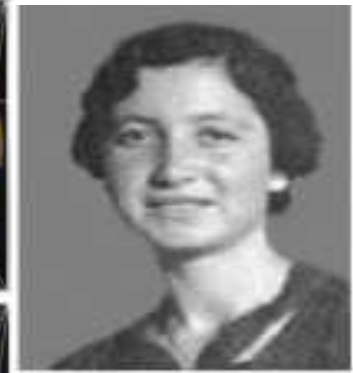

Begin-picture

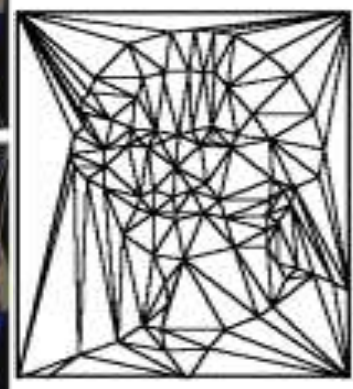

Begin-triangulation

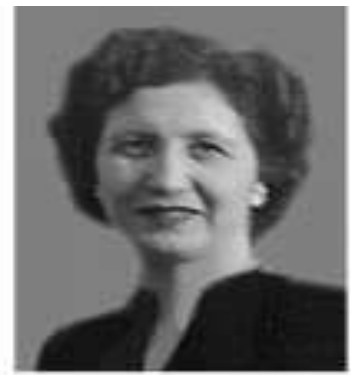

End-picture

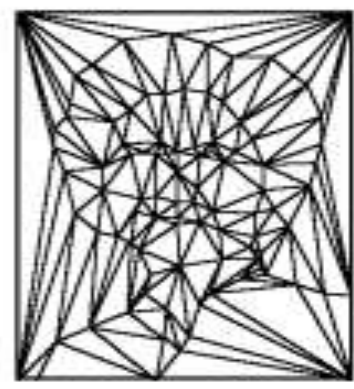

End-triangulation

Figure 3.3. The Process of Triangulation [Laden, 2011] [Anton et al., 2010]

In figure 3.3, the left set of images represents a sample frame sequence of 2 images that are being morphed. This sequence also depicts how each triangle is translated from image 1 to image 2 as part of the morphing process. In an image warp or morph, triangulation is a main component. Triangulation is the process of creating a maximum set of triangles from a set of points. When the points - the vertices of the triangles - are moved to new locations in the second image, the triangles are also transformed as a result of that movement. The left set of images depict the gradual decrease in thick sea ice in the arctic [Laden, 2011]. Abrosoft Fantamorph allows users to view the path the triangles overlaying the images take as the transformation from image 1 to image 2 occurs. The triangulation sequence allows one to view the linear movement of each point as it travels from its original location in the first image to its new location in the second image. The right set of images in figure 3.3 also depict the begin- and end- triangulation of two images [Anton et al., 2010]. Notice that though the dimensions of some of the triangles change because of the morphing process, all of the points and triangles stay within their immediate location so that they don't overlap. A perfect simple image warp/morph happens when the transition of the triangulation between image 1 (first/begin picture) and image 2 (second/end picture) is smooth and results in no overlaps or folds.

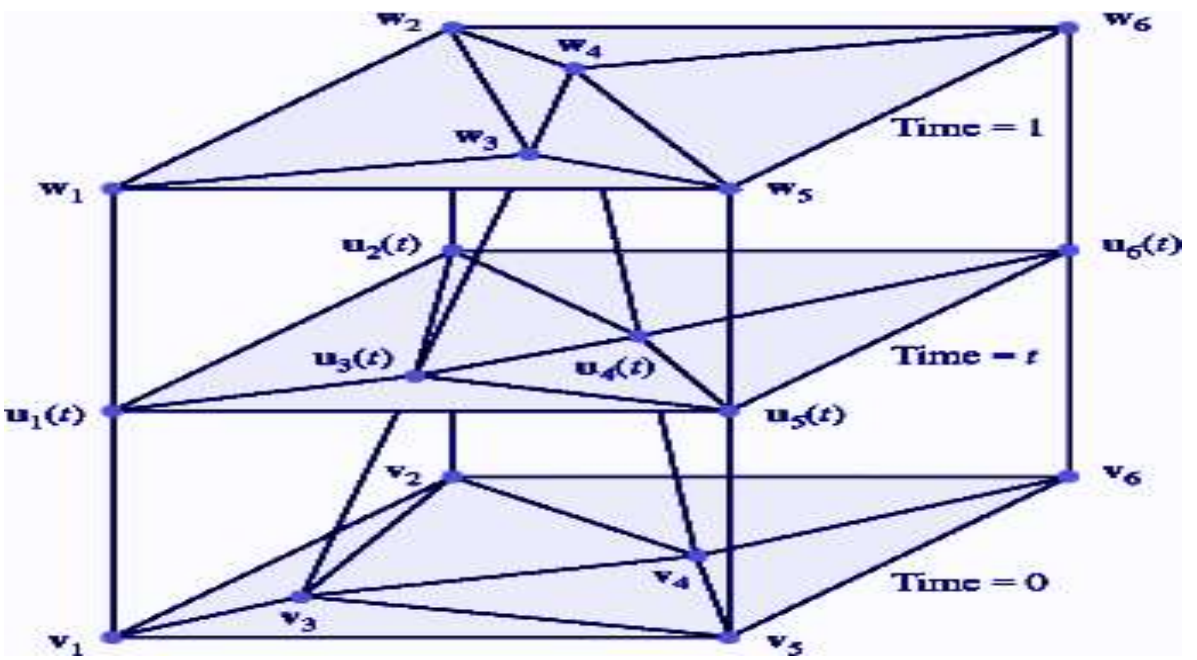

Figure 3.4. The Path of Triangulation During an Image Warp/Morph [Anton et al., 2010]

In addition to warping and morphing, there are other types of affine transformations. Below, more information about affine transformations and the mathematics behind them are outlined below. 
An affine transformation is any type of transformation that preserves co linearity (i.e., all points initially lying on a line still lie on a line after the transformation occurs) and the ratios of distances (e.g., the midpoint of any line segment on the image remains the midpoint after the transformation). Affine transformations base in linear algebra in which the transformation of an object follows a linear and constant pattern [Anton et al., 2010]. Computer graphics software and techniques allow for the manipulation and transformation of images in various ways - rotating, translating, shearing, scaling/dilating, cropping, slanting, etc [Anton et al., 2010]. In essence, any triangle is capable of being transformed into another triangle by the means of an affine transformation because all three vertices stay adjoined by three line segments. As a result, all triangle are affine. A general expression that relates source image locations to locations in the image transformed by an affine warp (or morph) is as follows [Anton et al., 2010]:

$$
\mathrm{P}_{\mathrm{S}}=\mathrm{A} * \mathrm{P}_{\mathrm{T}}
$$

Where:

$\mathrm{P}_{\mathrm{S}}=\left[\mathrm{x}_{\mathrm{S}} \mathrm{y}_{\mathrm{S}} 1\right]$; is the source location matrix

$\mathrm{P}_{\mathrm{T}}=\left[\mathrm{x}_{\mathrm{T}} \mathrm{y}_{\mathrm{T}} 1\right]$; is the corresponding location of the transformed image matrix

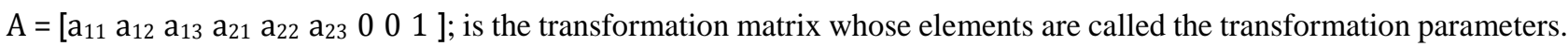

***Affine Transformation: Translation: A translation is a type of affine transformation in which only the general location of an image is changed. For example, if image 1 lies on a coordinate plane and the coordinates of its corners are $(0,1),(1,1),(1,0)$ and $(0,0)$, then, after a translation, the new coordinates might translate in $(1,1),(2,1)(1,0)$, and $(2,0)$. Everything about the images remains the same, except for its location.

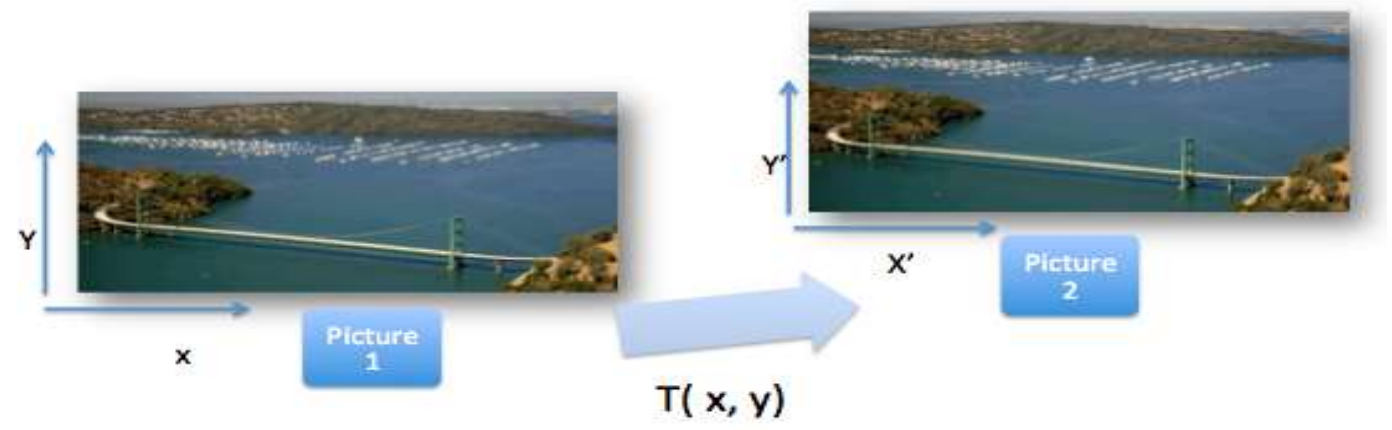

Figure 3.5. Translation Transformation [Spector, 2014]

Transformation Matrix: $A_{\mathrm{T}}=\left[\begin{array}{lllllllll}1 & 0 & d_{\mathrm{x}} & 0 & 1 & \mathrm{~d}_{\mathrm{y}} & 0 & 0 & 1\end{array}\right]$

Where $d_{x}$ and $d_{y}$ are the amounts of shift in $x$ and $y$ direction, respectively: $\quad d_{x}=p_{x}{ }^{\prime}-p_{x}$ and $d y=p_{y}{ }^{\prime}-p_{y}$

***Affine Transformation: Dilation/Scaling: Another type of affine transformation is a dilation (scaling). In a dilation, the size of the image changes so that it becomes larger or smaller. For example, if image 1 lies on a coordinate plane and the coordinates of its corners are $(0,1),(1,1),(1,0)$ and $(0,0)$, then, after a dilation (scaling) on a scale of two, the new coordinates of the image will be $(0,0),(0,2)(2,2)$, and $(2,0)$. The coordinates are multiplied by the dilation factor, which, in this case, was two. To increase the size of an image, multiply the coordinates by a dilation factor greater than one. Conversely, to decrease the size of an image, multiply the coordinates by a dilation factor that is less than one.
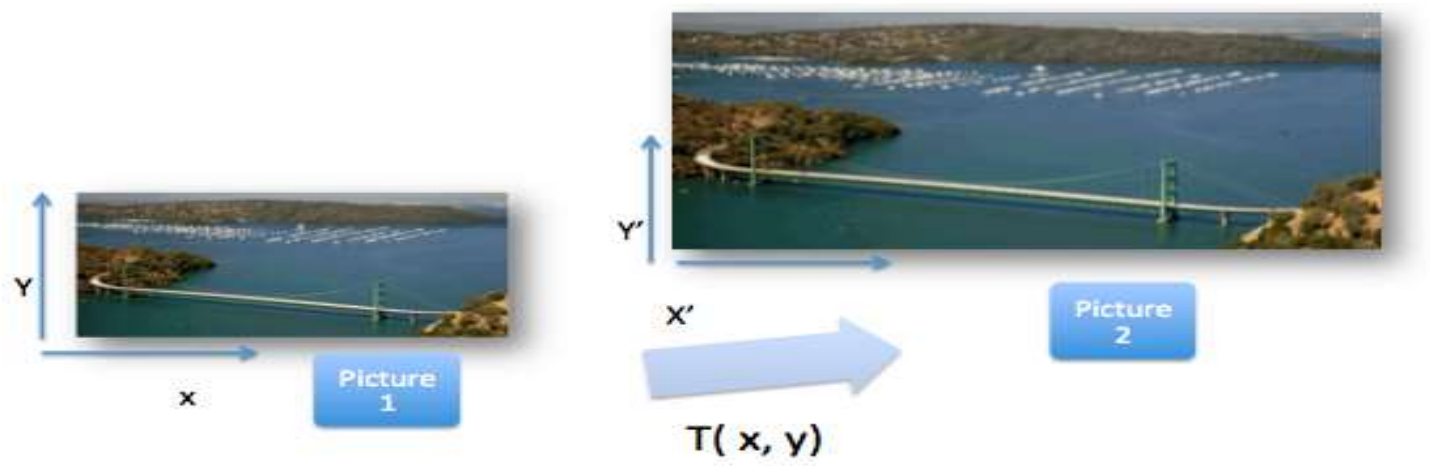

Figure 3.6. Dilation/Scaling Transformation [Spector, 2014] 
Transformation Matrix: $A_{s}=\left[\begin{array}{lllllllll}\beta_{\mathrm{x}} & 0 & 0 & 0 & \beta_{\mathrm{y}} & 0 & 0 & 0 & 1\end{array}\right]$

Where $\beta_{\mathrm{x}}$ and $\beta_{\mathrm{y}}$ are the scale/dilation factors along the $\mathrm{x}$ and $\mathrm{y}$ directions, respectively.

***Affine Transformation: Rotation: A rotation occurs when the image is rotated at a specific angle from a specific point. When undergoing a 180 degrees rotation from anyone of its corners, an image is flipped upside down. After a 360 degrees location from any corner, the image arrives back in its original location. The matrix for this type of affine transformation involves trigonometry.
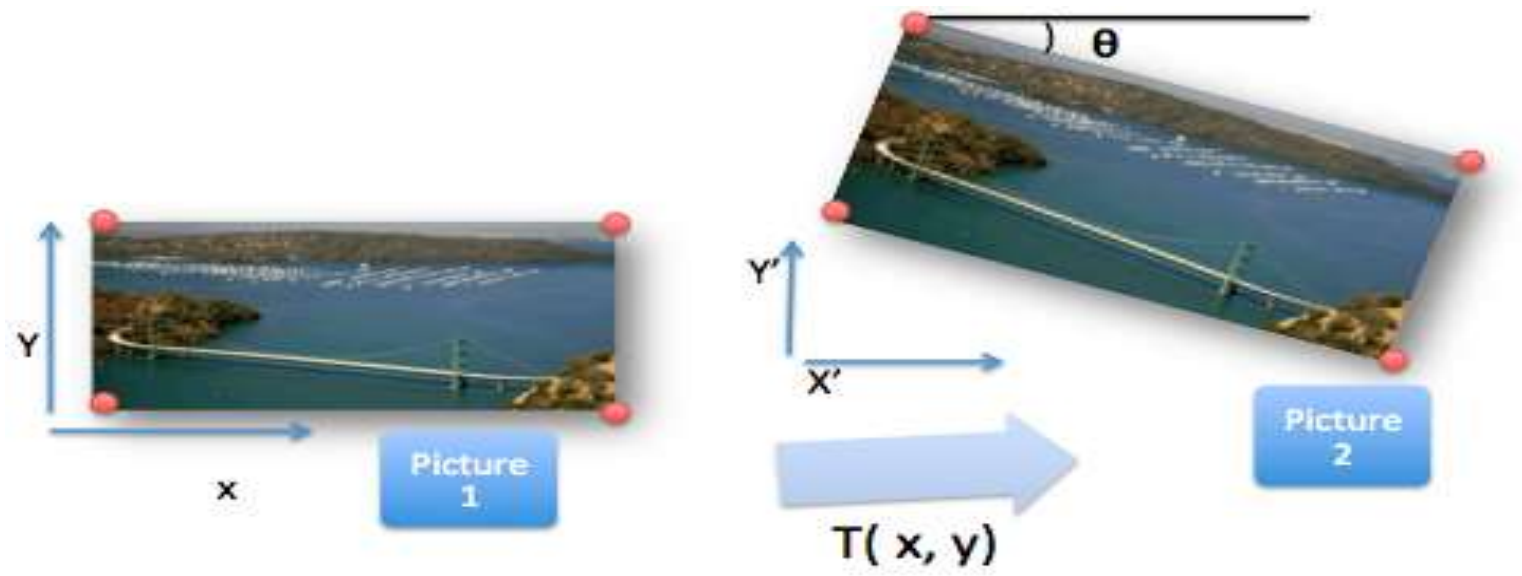

Figure 3.7. Rotation Transformation [Spector, 2014]

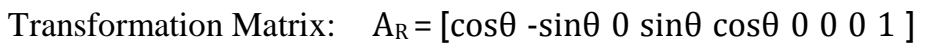

Where $\theta$ is the angle of rotation.

***Affine Transformation: Shearing: Image shearing is a special type of an affine transformation in which one part of an image is shifted towards a specific direction and another part of the image is also shifted to a different direction. For instance, when horizontally shearing a square image, the upper right part is shifted more to the right while the lower left part shifts more to the left. In this case, the square shape of the original image is transformed into a diamond shape.
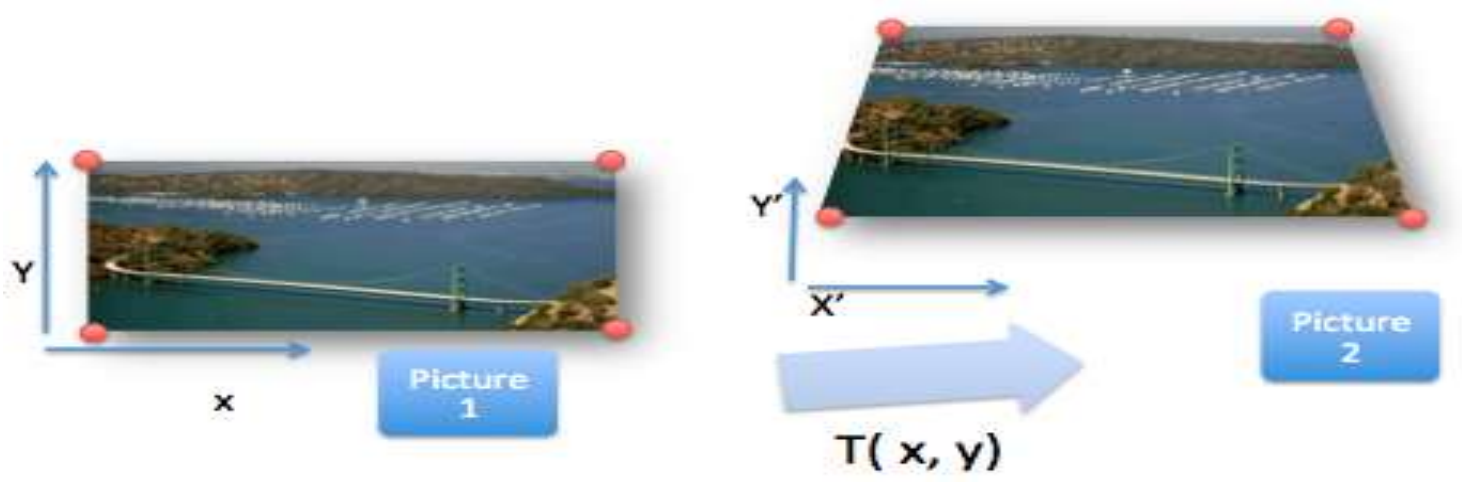

Figure 3.8. Shearing Transformation [Spector, 2014]

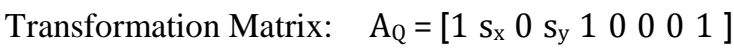

Where $s_{x}$ and $s_{y}$ are parameters for shearing along $x$ and $y$ axes.

\section{Discussion and Future Research}

After analyzing the various warps and morphs that the team generated using the Abrosoft FantaMorph software, it is reasonable to conclude that computer warping and morphing techniques are indeed incredibly useful in an array of fields. Whether it is the mapping of global climate change or simply the 3D representation of a planet, these techniques play an 
essential role in creating depictions of important concepts and bettering our understanding of change - from the past to the present to the future. At the conclusion of this study, it is clear that the mathematics behind warps and morphs have a direct connection to how these techniques are translated to images and that both of these computer imaging techniques can be applied to a plethora of fields and disciplines.

Though these techniques have quite a number of useful applications, unfortunately, they are also comprised of a small number of limitations. For example, because some morphs can only be instigated in a linear fashion and the change between each frame in the morph happens at a constant rate. As a result, the gradual process that an object undergoes as it transitions from the first image to the second image may not be accurately represented. Numerous natural processed to do not happen in a linear, constant pattern, so it would be inaccurate to presume that morphs created of the images depicting hose processes are error-free. To remedy this, software that can morph objects in trigonometric or quadratic fashions might be useful. Furthermore, an additional major shortcoming the team members experienced while generating morphs was overlapping triangles during the relocation of points. If an object undergoes dramatic changes in which it loses or gain new parts, it is inevitable that the triangles will overlap at some point of the morphing process. As a result, it is simply impossible for some objects to be morphed between images. One of the only methods to resolve this problem is to divide the complete image of the object into smaller images and consequently, morph each part of the image separately. Finally, one would collate all the separate morphs together to run them at the same time. Though there are more possible limitations of using image warping and morphing techniques, these two are of paramount importance.

In order to take this research further, teams can analyze the functions of warps and morphs that do not work in linear fashions. While this study analyzes the mathematics and applications of the most simple type of morph - linear morphs (in which image frames morph at a constant rate) - it is also crucial to study the mathematics and applications behind other varieties of warping/morphing techniques in order to gain better understanding and depictions of objects that do not transform in linear manners. Moreover, further research can also concentrate on the roles of warps and morphs in creating projections. This research would play a significant role in assisting scientist and organizations, such as NASA, to predict future occurrences.

\section{Relevance to the National Aeronautics and Space Administration}

The National Aeronautics and Space Administration can use different warping and morphing techniques for a plethora of reasons: to depict environmental changes on Earth, to create better representations of extraterrestrial objects by transforming 2D satellite images into 3D virtual camera views, to combine images captured by different satellites to form more uniform and cohesive pictures of objects, to depict the gradual process that an object undergoes within the span of time the two images are taken, to emphasize any slight or easily overlooked changes an object experiences, to collate images that depict a single object with respect to time, and more. Distorting images can also be used to create projections. For example, NASA can warp an image of a map of Florida by manipulating the coastline in accordance to how scientists predict the coast of Florida will look like in a few decades due to inevitable sea level rise. Morphing techniques can depict how this process will look like over the specific span of time corresponding to this projection. Furthermore, NASA can create 3D virtual camera views of planets such as Mars and Pluto by morphing 2D photographs of these planets taken by satellites at different angles. This morphing technique will allow NASA to obtain more cohesive depictions of these planets and other extraterrestrial objects. The beauty of warping and morphing techniques is that they have an impressive array of applications and uses for the purpose of exploring change and depicting objects.

\section{Acknowledgements}

- National Aeronautics and Space Administration (NASA);

- $\quad$ NASA Goddard Institute for Space Studies (GISS);

- NASA Goddard Space Flight Center Office of Education;

- CUNY Hostos Community College;

- CILES Continuous Learning;

- National Oceanic and Atmospheric Administration (NOAA);

- United States Department of Education;

- Grant from the US Department of Education: \# P031C110158;

- National Science Foundation;

- $\quad * * *$ Special Thanks to Prof. Angulo Nieves 


\section{References}

Abrosoft Fantamorph 5.

Anton, H., \& Rorres, C. (2010). Elementary linear algebra: Applications version. (10th ed.). John Wiley \& Sons, Inc.

Climate Discovery. (2013, April 14). Dangerous record ozone hole reporting. Retrieved from http://climatediscovery.com/dangerous-record-ozone-hole-reporting/

Durand, F., \& Freeman, B. (n.d.). Image warping and morphing. Retrieved from http:/groups.csail.mit.edu/graphics/classes/CompPhoto06/html/lecturenotes/14_WarpMorph_6.pdf

imgLOP. (2015). Cancer; normal. Retrieved from http://imglop.com/img/aHR0cDovL2ltdXNOdHJhdmVsLmNhL3dwLWFkbWluL2NhbmNlci1jZWxscy1hbmQtb m9ybWFsLWNlbGxzLWRpZmZlcmVuY2UtaTEwLnBuZw==

Laden, G. (2011, October 10). Global warming is melting the ice caps. Retrieved from http://scienceblogs.com/gregladen/2011/10/10/gobal-warming-is-melting-the-i/

Morpheus Photo Morpher.

NASA. (2013, September 4). What is a supernova?. Retrieved from http://www.nasa.gov/audience/forstudents/5-8/features/nasa-knows/what-is-a-supernova.html

Null-Entity. (n.d.). 360 head turn around. Retrieved from http://orig12.deviantart.net/9e4c/f/2013/134/5/8/360_head_turn_around_by_null_entity-d659y7s.jpg

Park, A., \& Lurie, J. (2014, February 27). Sorry, california. a little rain isn't going to save you. Retrieved from http://www.motherjones.com/blog/2014/02?page=2

Schindler, T. (2014, June 26). New NASA Images Highlight U.S. Air Quality Improvement. Retrieved from https://www.nasa.gov/content/goddard/new-nasa-images-highlight-us-air-quality-improvement/\#.Vco5cM64neQ

Seitz, S., \& Dyer, C. (2000, June 29). View morphing. Retrieved from http://homes.cs.washington.edu/ seitz/vmorph/vmorph.htm

Spector, D. (2014, February 27). 6 Crazy Photos That Show Why California Is Desperate For Rain. http://www.businessinsider.com/photos-of-california-drought-2014-2\#ixzz3h7DFv9NV

World Regional Geography: People, Places and Globalization. (n.d.). Regions of the united states and canada. Retrieved from https://saylordotorg.github.io/text_world-regional-geography-people-places-and-globalization/s07-05-regions-of-th e-united-states-a.html

(n.a.). (n.d.). Andy Roane. Retrieved from www.workforce.com and http://www.workforce.com/ext/resources/PEOPLE-MOVES-Andy-Roane.jpeg

\section{Copyrights}

Copyright for this article is retained by the author(s), with first publication rights granted to the journal.

This is an open-access article distributed under the terms and conditions of the Creative Commons Attribution license (http://creativecommons.org/licenses/by/3.0/). 